squamous cell carcinomas in a patient treated by photochemotherapy for

17 Klepp O, Magnus K. Some environmental and bodily characteristics of melanoma patients. A case-control study. Int $\mathcal{f}$ Cancer 1979;23:482-6.

18 Adam SA, Sheaves JK, Wright NH, Mosser G, Harris RW, Vessey MP. A case-control study of the possible association between oral contraceptives and malignant melanoma. Br $\mathcal{C}$ Cancer 1981;44:45-50.

19 Elwood JM, Gallagher RP, Stapleton PJ. No association between malignant melanoma and acne or psoriasis; results from the western Canada melanoma study. Br F Dermatol 1986;115:573-6.

20 Holly EA, Kelly JW, Shpall SN, Chiu S-H. Number of melanocytic nevi as a major risk factor for malignant melanoma. I Am Acad Dermatol 1987;17:459-68.

21 Smith PG, Pike MC, Hill AP, Breslow NE, Day NE. Multivariate conditional logistic analysis of stratum-matched case-control studies. Applied Statistics 1981;30:190-7.

22 Brown AP. Melanoma and fluorescent light. Lancet 1982;ii:1398.

23 Larkö O, Diffey BL. Natural UV-B radiation received by people with outdoor, indoor, and mixed occupations and UV-B treatment of psoriasis. Clin Exp Dermatol 1983;8:279-85.

24 Devgun MS, Johnson BE, Paterson CR. Tanning, protection against sunburn and vitamin D formation with a UV-A 'sun-bed'. Br $\mathcal{I}$ Dermatol 982:107:275-84.

25 Nachtwey DS, Rundel RD. A photobiological evaluation of tanning booths Science 1981;211:405-7.

26 Swart R, Kenter I, Suurmond D. The incidence of PUVA-induced freckles. Dermatologica 1984;168:304-5.

27 MacPherson TD, Finlay AY. Ultraviolet A freckles: another hazard of sunbeds? Br Med f 1986;292:380.

28 Stern RS, Thibodeau LA, Kleinerman AB, et al. Risk of cutaneous carcinom in patients treated with oral methoxsalen photochemotherapy for psoriasis. N Engl f Med 1979;300:809-13.

29 Hersey P, Bradley M, Hasic E, et al. Immunological effects of solarium exposure. Lancet 1983; i:545-8.

30 Proctor MS, Cox AJ, Grais LS. Lentigo maligna melanoma in a treated psoriatric plaque. Arch Dermatol 1981;117:149-50.

31 Beral V, Evans S, Shaw H, Milton G. Cutaneous factors related to the risk of malignant melanoma. Br I Dermatol 1983;109:165-72.

32 Alderson MR, Clarke JA. Cancer incidence in patients with psoriasis. Brf Cancer 1983;47:857-9.

(Accepted 12 May 1988)

\title{
Evaluation of a proposed breast cancer screening regimen
}

\author{
E G Knox
}

\begin{abstract}
The Forrest working group on breast cancer screening recommended routine mammography for women in the United Kingdom at ages 50, 53, 56, 59, 62, and 65. Benefits were costed at about $£ 3000$ for each life year recovered, but there was no estimate of the cost of each life saved, and the consequent reduction in mortality from breast cancer in the general population of the United Kingdom was not estimated.

The present study addressed both of these issues using an interactive computer modelling process. Long term savings were calculated at 900 deaths a year in England and Wales-that is, about $8 \%$ of the total deaths from breast cancer-and $9 \%$ of life years currently lost. The cost of each death saved from breast cancer was estimated at $£ 39000$.
\end{abstract}

\section{Introduction}

A recent report on screening for breast cancer by the Forrest working group ${ }^{1}$ recommended introducing mammography screening in women at intervals of three years between the ages of 50 and 65 years-that is, six screens altogether. ${ }^{1}$ The age recommendation was based partly on an interpretation of earlier studies that screening is ineffective in women aged under $50^{2.5}$ and partly on the observation that older women are reluctant to attend. Economic appraisals based on projected savings of life years were considered to be favourable.

The report did.not, however, predict the long term reductions in mortality among the total population. It did not clearly distinguish between true mortality in the population and the serial cumulative differences in mortality observed in trials. Nor did the report give estimates of the cost of each death from breast cancer avoided. This paper examines these questions.

\section{Methods}

Computer based methods for predicting the outcomes of population screening for cancer were worked out many years ago, and early predictions of the effectiveness of screening for breast cancer in the United Kingdom were reported in $1975 .{ }^{67}$ The calculations were based on, firstly, age specific distributions of mortality from (or incidence of) cancer in the population; secondly, a prior knowledge or reasonable estimate of the duration and rates of progression of the different stages of the disease; and, thirdly, the sensitivity and specificity of the test, the therapeutic efficacy of the treatment, the choice of ages at which to screen, and the expected acceptance rates for specific ages.

The chief problems of applying the predictions stemmed from uncertainties about the clinical course of the early stages of cancer, and this had been a particular problem in the case of cervical cancer, as the diagnosis (by cone biopsy) itself destroyed the lesion, precluding subsequent observation of its progress. A simplified predictive method which circumvented the worst of these problems was therefore developed. ${ }^{8}$ It depended on specifying the clinical course only in cases which progressed to a particular endpoint which it was the aim to prevent-for example, death. Lesions that did not progress were not considered at all. This had disadvantages - for example, we could not then comment on the numbers of false diagnoses or unnecessary treatments. For the preventive target group, however, a clear cut relation between the premises and the outcomes could be established.

The clinical course of the target disease (for example, breast cancer) is first conceived of as comprising two non-varying periods-namely, A and B. During period $A$ the disease is susceptible to early detection and full or partial cure. In period $B$ the disease is incurable. When screening is offered at a particular age and the offer is maintained over several years it results in a subsequent dip in the mortality curve at later ages. Figure 1 illustrates this. The delay between the

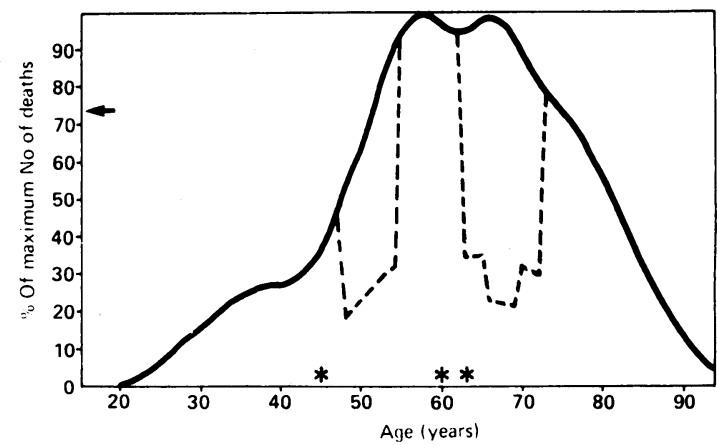

FIG 1-Method of simulation showing effects of single screen at age 45 and of two screens with overlapping "domains" at ages 60 and 63. $\star$ Screening ages and subsequent age distribution of absolute numbers of deaths. $---=$ Number of deaths after screening. $\leftarrow=$ Reduction in mortality owing to screening. Angular curves reflect fact that in this demonstration phases of clinical course were unvarying between different ages and unvarying between different women of given age. Period $A=6$ years. Period $B=3$ years 
start of screening and the dip in mortality is due to women who have already entered period B when screening begins, who will die within B years (three years in this example). Screening offers them nothing. The width of the dip corresponds to period A and is caused by reduced mortality in women whose disease was partially or fully curable at the time of screening. The position and duration of the dip are thus obtained by setting the clinical course of the disease across the graph from left to right in reverse: period $B$ is entered first (starting from the age of onset of screening), which gives the lag period before the start of the dip; then period A, which gives the width of the dip. The depth of the dip is the product of the rate of acceptance, the sensitivity of the test, and the efficacy of treatment. If periods $A$ and $B$ are variable then the angular shape of the dip in figure 1 is modified (shown in fig 2).

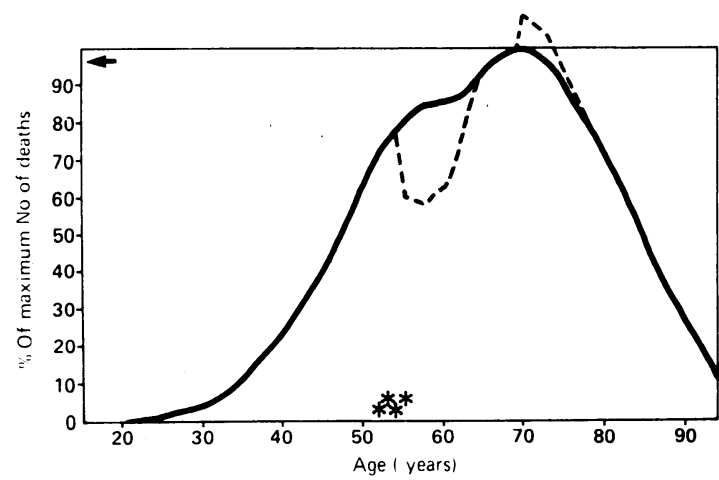

FIG 2-Simulation of Health Insurance Plan Study. ${ }^{*}$ Screening ages in "treated" group. $-=$ Deaths from breast cancer in UK. ----= Number of deaths in "treated" group after screening. $\leftarrow=$ Reduction in mortality owing to screening. Period $A=3$ years. Period $B=4$ years

Earlier versions of the model supposed that the clinical course was constant with age,${ }^{8}$ but the present version allows variation in this respect. Allowance can also be made for increasing sensitivity of screening tests across period $\mathrm{A}$ and for diminishing efficacy of treatment. Rates of acceptance can be declared for different ages. Outcomes can be expressed in terms of savings of deaths or savings of life years. Each of these can be expressed for each 1000 tests delivered. The computer based system incorporating these facilities allows detailed prescription of the screening regimen; alternatively, it allows the user to declare the number of tests offered and itself optimises the age distribution. Both procedures were used in the following application to cancer of the breast.

The target group was identified as women who die from breast cancer in England and Wales. The age distribution was obtained from reports of the Office of Population Censuses and Surveys and figures 2-4 show its form. This is a distribution of absolute numbers of deaths (rather than rates) on a simple arithmetic scale,

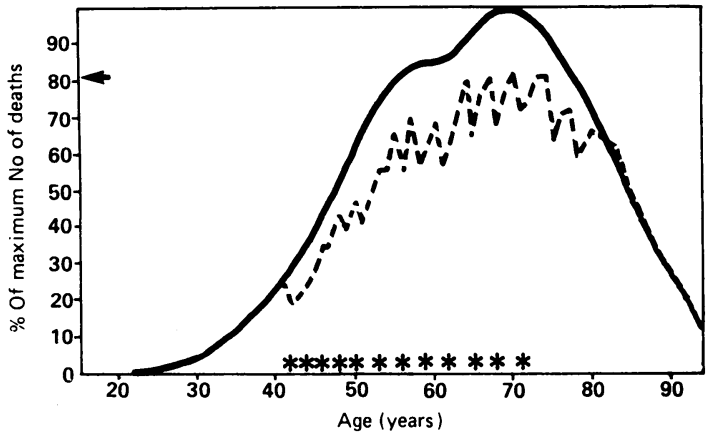

FIG 3-Simulation of Swedish Two Counties study. * Screening ages in "treated" group. - Deaths from breast cancer in UK. - - = Number of deaths in "treated" group after screening. $\leftarrow$ Reduction in mortality owing to screening. Period $A=3$ years. Period $B=4$ years

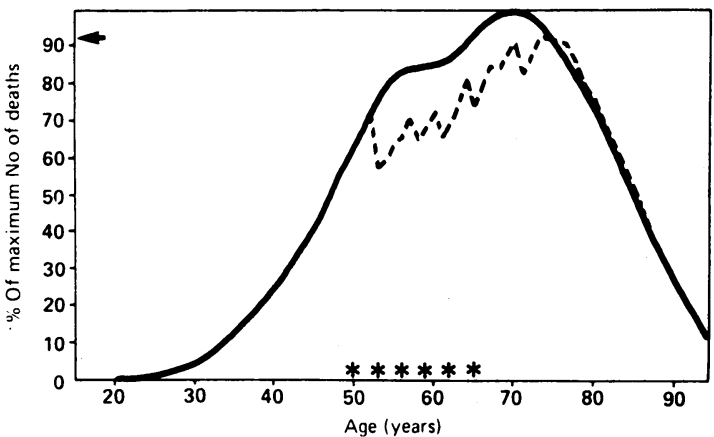

FIG 4-Simulation of deaths saved under Forrest proposals. ${ }^{\star}$ Screenin ages proposed. $-=$ Deaths from breast cancer in $U K .-\ldots=$ Number of deaths in "treated" group after screening. $\longleftarrow=$ Reduction in mortality owing to screening. Period $A=3$ years. Period $B=4$ years

so it supplies a clear picture of where the problem lies and where the benefits might be obtained. The duration of the clinical stages, the performance measures of the screening procedure, the efficacy of treatment in cases detected by screening, and the likely rates of acceptance at different ages were obtained by referring to published material. This information is seldom available in the exact format required by the model, so the various measures were developed in two stages. Directly relevant data were first inspected and abstracted. The estimates were then refined through "fitting" the predictions of decreases in mortality to the results obtained from two well conducted randomised trials - namely, the New York Health Insurance Plan study $^{2-4}$ and the Two Counties study from Sweden. The fitting process went through several cycles until the life saving outcomes of both studies could be matched. The refined measures were then used to simulate the recommendations of the Forrest working group and to predict the outcomes of its proposals. Finally, the marginal effects of incremental increases in resources and of different age deployments were measured.

\section{CLINICAL COURSE}

Estimates of the durations of stages A and B were obtained from the Two Counties study. Differences in mortality between cases and controls first appeared four years after the trial was started. We may take this interval to correspond to the duration of stage $B$. The greatest case-control differences occurred between five and seven years after the beginning of the programme, giving a dip width of three years, corresponding to stage $A$. The working estimates for stages $A$ and $B$ were therefore three years and four years, respectively, or seven years' total clinical course at around age 55 . The results of the Health Insurance Plan investigation fit reasonably with this figure, though the additional use of palpation in screening makes estimating the effect of mammography alone difficult. Maintaining the same case-control differences into later years in the Health Insurance Plan study supports the reliability of the estimates, ${ }^{4}$ so these "average" values for stages A and B were held constant throughout the subsequent investigations. As the duration of the clinical course of the disease is almost certainly shorter among young women than old, however, the combined period of seven years at age 55 was set to vary from 5.4 years at age 25 to 8.6 years at age 85 . These are mean values, and additional variation was introduced at each individual age - thus at age 55 the total duration of the clinical course was varied between $4 \cdot 2$ years and $9 \cdot 8$ years, with equivalent variations at other ages.

The "average" durations of the clinical course can be regarded as median points that half of the women have passed within this time while the other half have yet to do so. In practice, experiments using different degrees of variation, both within ages and between ages, made 
little difference to fittings of outcome or to any of the subsequent results.

\section{SENSITIVITY AND THERAPEUTIC EFFICACY}

The Swedish investigation suggests a mammographic sensitivity of about $0.7(70 \%)$ if we count as missed cases any that occurred within three years of the cancer screening. This is rather greater than that for the mammography element of the Health Insurance Plan study, though the combined use of mammography and palpation ${ }^{2}$ makes comparison difficult. Changes in technique suggest that we should accept the estimate from the more recent Swedish investigation In the Two Counties study the interval between screens was three years, so this estimate of sensitivity can be taken to correspond with the middle of period $\mathrm{A}$, which is also of three years' duration. For breast cancer the sensitivity will presumably rise as period A elapses, while cure rates will fall. Arbitrarily (but with adequate consequences for fitting), sensitivity was set to vary between 0.2 at the beginning of period $A$ and 0.9 at the end. The cure rate was set to 0.55 in the middle of period $\mathrm{A}$, declining from 0.9 at the beginning to 0.2 at the end. At each stage $35 \%$ of the remaining women were considered to be partly cured, with a lifespan extension of 15 years. Further justification of this choice of variables follows.

\section{CHOICE OF VARIABLES}

Some of the variables adopted were arbitrary. In addition, the choice of one often to some extent depended on the choice of another. In practice they could not be derived in a single step but were developed and refined through a series of iterations in which the Health Insurance Plan and Swedish trials were mimicked and in which the variables were adjusted until the numbers of women saved from death were successfully matched.

The circumstances of the trials differed. The Health Insurance Plan study was simulated with four screens at ages 52, 53, 54, and 55 (fig 2). The level of attendance was set at $55 \%$, a compromise between the $63 \%$ who initially accepted and the $44 \%$ who completed the course of four screens. The Swedish simulation (fig 3) was based on screening at intervals of three years between the ages of 50 and 71 years and at intervals of two years between the ages of 40 and 50 years, as in the trial itself. Compliance was set at $90 \%$ at age $40,85 \%$ at age 60 , and $70 \%$ at age 80 , with linear interpolations. This too corresponded with the report of the trial itself.

Levels of compliance, patterns of age deployment, and total investments differed between the trials and were represented appropriately in the different simulations. The two simulations used a common set of measures of clinical course and sensitivity as stated above. On this basis a common set of estimates of therapeutic efficacy was also developed, making repeated adjustments until calculated savings converged with those observed, allowing for the different ways in which they were reported.

In the Swedish simulation age specific mortality became established at a new steady level 10 years after the start of the study. By this time the simulated cumulative mortality across the age band $43-73$ years had fallen by $28 \%$, which compares well with the fall of $30.4 \%$ after seven years observed in the actual study (fig 3).

The simulation of the Health Insurance Plan trial addressed a narrower initial age band, effectively a cohort with a median age of 54 years, but followed it for longer, mimicking the real study. The decrease in age specific mortality resulting from screening began at 58 years (six years after the onset of screening) and was complete by 65 years, with a small rebound between 70 years and 76 years as the survivors who had partial cures came to the end of their extended life-span (fig 2). The maximum decrease in simulated cumulative mortality in the screened group was $27.4 \%$ below the mortality of unscreened cases. In the trial itself the maximum percentage difference was obtained after five years, and the cumulative mortality among the screened was then $38 \%$ below that of the unscreened cases, ${ }^{4}$ but this result was based on palpation as well as on mammography, which probably accounts for the greater effect. The difference in simulated cumulative mortality diminished to $12 \%$ after 20 years, in line with the reduced numbers of new cases that would be identified after the high initial rate on first screening. In the trial itself the mortality difference was $17 \%$ after 14 years.

\section{DIFFERENCES}

The differences in outcome between the Swedish and New York studies probably stemmed from the different trial designs rather than from fundamental differences in the efficacy of the tests or the treatments. For example, the design of the Health Insurance Plan study benefited disproportionately from the high rates of detection associated with first attendances and from the combination of palpation and mammography in the screening procedure.

The cumulative differences in case-control mortality after intervention that were used in each study as criteria for judging outcome are not true population measures. They cannot be defined independently of the particular design and implementation, and they cannot be transferred directly from one circumstance to another. For example, the true saving in mortality from the Health Insurance Plan pattern if it were adopted as a long term intervention policy in the United Kingdom would amount to only $3 \cdot 6 \%$ of all deaths from breast cancer, while the Swedish pattern similarly applied would result in a saving of $19 \cdot 6 \%$.

The benefits of both could be improved in use by increased investment or by a more efficient use of an equivalent investment. For example, the designers of the Swedish trial shortened the intervals between screening in the age bands where the clinical course was thought to progress more rapidly. This is logically dubious. For any limited resource it is usually better to concentrate the screening effort on ages when the prevalence of curable disease is greatest. This normally occurs shortly before the age of maximum mortality and at ages when the clinical course is most drawn out. A simulated age deployment optimised by the computer program did exactly this, raising the expected benefit from the same investment to $23 \%$ of (British) deaths from breast cancer.

\section{Results}

\section{THE FORREST PROPOSALS}

The Forrest group hoped that a general mammography service in the United Kingdom might attract an attendance of about $70 \%$. This is far beyond the attendance rates generally obtained in the same age groups for cervical cancer screening in the United Kingdom, and it is wishful thinking to suppose that results obtained in special studies can be matched in more general applications. Even the trials vary widely. The Swedish investigation attained $83 \%$ attendance, but in New York the initial attendance was $63 \%$ and the full course of four screening examinations was completed by only $40 \%$. In the Netherlands $72 \%$ attendance was attained for initial screening and in Edinburgh $63 \%$. In Guildford the response to a first invitation was $69 \% .^{\prime}$ Attendances after recurring invi- 
tations are not yet available for all these studies, but are likely to be lower.

The Forrest proposals were simulated in the computer model. For the purposes of this simulation the screening ages were set at $50,53,56,59,62$, and 65 years. Compliance was set at $60 \%$ at 40 years, $50 \%$ at 60 years, and $40 \%$ at 80 years, with linear interpolations. Figure 4 shows the predicted long term results in terms of lives saved, and figure 5 shows the savings of life years. The full programme of procedures would save

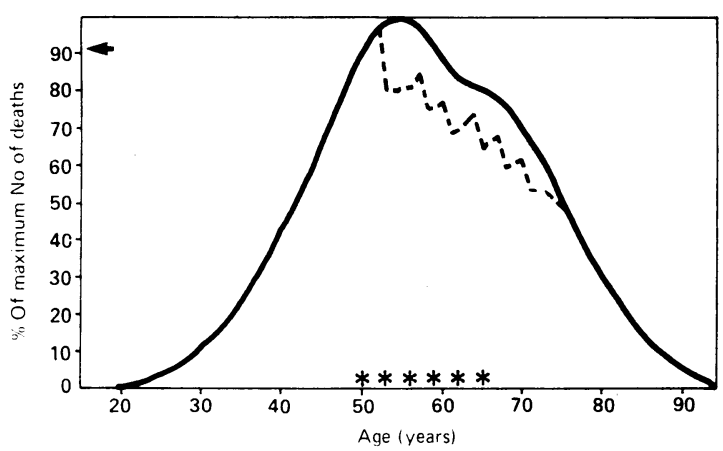

FIG 5-Simulation of life years saved under Forrest proposals. ${ }^{\star}$ Screening ages proposed. $-=$ Life years lost from breast cancer in UK. - - - =Number of life years saved in "treated" groups after screening. $\leftarrow=$ Reduction in lost life years owing to screening. Period $A=3$ years. Period $B=4$ years

$7 \cdot 6 \%$ of all deaths from breast cancer and $8 \cdot 6 \%$ of the life years lost. It would save 1.06 deaths and 24.7 life years for every 1000 tests performed. Total lives saved would amount to 901 from a target group total of 11877 (England and Wales 1978).

\section{ALTERNATIVE DEPLOYMENTS AND ALTERNATIVE RESOURCES}

The program allows the user to specify the number of tests to be offered and itself optimises the age distribution. Optimisation can be based on the number of deaths saved, the number of life years saved, or the ratio between either of these and the number of tests performed. Optimisation of the investment proposed by Forrest, in terms of deaths saved, resulted in a wider age deployment than was recommended. Optimised ages ranged from 51 to 71 , and the deaths saved in England and Wales rose from 901 to 1194 , or $10.1 \%$ of the target group. Optimisation for life years saved resulted in an age distribution ranging from 41 to 64 years. The number of life years saved then rose from $8 \cdot 6 \%$ to $11 \cdot 5 \%$ of life years otherwise lost. The first exercise increased operational efficiency to 1.48 deaths and 29.6 life years saved for every 1000 tests; the second exercise resulted in $1 \cdot 12$ deaths and 30.9 life years saved for every 1000 tests. These improvements are quite modest, and the simulated outcomes (figs 4 and 5) of the Forrest recommendations themselves do not fall far short of them. The proposals therefore represent a reasonable compromise between a desire to save lives and a desire to save life years. Not much is to be gained from redeployment.

Next, a series of simulation experiments was undertaken in which successive increments of investment were supplied. Each increment was optimised based on the criterion of life years saved, and the marginal benefits of each increment were measured. The pattern showed the familiar curve of diminishing returns. For example, introducing two tests at ages 50 and 57 recovered $4.8 \%$ of life years otherwise lost, but increasing the number of tests from 12 to 14 saved only an additional $2 \cdot 7 \%$ of life years.

The pattern of diminishing returns represents the progressive stripping of age groups that show a high yield, and the enforced overlap of test domains. It also depends on the manner in which the slower growing tumours are preferentially intercepted. The model copes with these effects, but accurate projection beyond the investment level of the Two Counties study would probably require a more detailed knowledge of heterogeneities in the clinical course than we can presently command. The curve also depends on the proportion of women who refuse to attend for any test. To mimic adequately larger investments we would need to stratify the population according to attendance patterns and run the model separately for each layer. For simulated increments within the investment levels provided in Sweden, however, the calculation can be regarded as being reliable and leads to the conclusion that the particular investment of resources proposed by the Forrest group has no intrinsic justification (as the group itself recognised). The simulation experiments seem to show that we could invest at least twice as much before encountering seriously reduced efficiency.

\section{Discussion}

The principle of representing the durations of the stages of the clinical course in reverse, beginning from the point of termination, is unfamiliar to clinical practitioners. They generally associate formulations of the clinical course with prognosis and tend to think prospectively. The principle, however, follows logically as soon as we nominate a terminal preventive target group and as soon as we accept the objective of intercepting the process leading to this outcome.

Estimates of the performance variables of the intervention also raise conceptual and practical difficulties. The different variable declarations are dependent on each other. Thus the values adopted for "sensitivity" and "therapeutic efficacy" depend on the duration of the phase of the clinical course for which the possibility of detection is contemplated. For these reasons the variables for this model must be assembled as a package and developed jointly through fitting the outcomes of their joint formulation to observations made during trials. We were fortunate in having the results of two well conducted experiments.

Extrapolations beyond the immediate vicinity of the "fitting" must be interpreted cautiously. It helps in this case that the investment proposals for the United Kingdom are intermediate to those of the New York and Swedish investigations. Simulated outcomes fitted to these studies can therefore be used with some confidence. The simulated curve of diminishing returns for increasing investment also provides reasonable guidance within the range set by these other studies. It seems that we could double the recommended investment and expect reasonable marginal returns for the extra costs.

The graphical displays clearly show the relation between the serial cumulative differences in mortality after exposure to screening as measured in a trialloosely and sometimes misleadingly designated simply as "mortality"- and true changes in mortality from breast cancer in the general population. The model shows that the benefit to be expected in Britain from a general implementation of the Forrest proposals, at efficiencies reached in trials, is a saving of about $7 \cdot 6 \%$ of mortality from breast cancer. This is much less than the $30 \%$ that a direct transfer of the observed mortality differentials in trials might seem to indicate. The difference between the two measures has been recognised for many years, ${ }^{7}$ but misinterpretations still occur. The estimated saving nevertheless amounts to 900 deaths a year in England and Wales, and the attempt is probably justified.

The findings have implications for the manner in which we might monitor the effectiveness of the new service. Eventual reductions of $8 \%$ in mortality in 
individual districts, each currently with about 60 deaths from breast cancer a year, would scarcely be measurable in the short to medium term, even if an efficient deployment were immediately attained. It would therefore seem sensible to conduct sequential and comparative evaluations for groups of 10 or 20 health districts rather than for one or two.

The working group calculated a cost of $£ 3000$ to $£ 3400$ for one life year saved, at 1985-6 prices. The exact workings are not easy to follow, but a parallel estimate is possible. The report estimates annual running costs of about $£ 18$ million plus amortisation on a capital investment of $£ 31$ million-say $£ 25$ million overall. The model estimate of 901 deaths and 21065 life years saved in England and Wales therefore suggests a non-discounted cost of $\$ 27747$ for each death saved and $\$ 1176$ for each life year saved. Discounting at $5 \%$ a year (as used by Forrest) between the year of the investment and the year of the benefit brings the cost of each life year saved to $£ 2591$, rather better than the Forrest estimates. ${ }^{1}$ The report does not give an estimate of costs for each death from breast cancer saved, but discounting our own estimate at the same rate over seven years gives a corresponding value of $£ 39043$.

This work was carried out as part of a health services research programme supported by the Department of Health and Social Security.

\section{Forrest P. Breast cancer screening. London: HMSO, 1986. \\ Shapiro S, Strax P, Venet L. Periodic breast cancer screening in reducing mortality from breast cancer. $7 A M A$ 1971;215:1777-85. \\ 3 Shapiro $\mathrm{S}$. Evidence on screening for breast cancer from a randomised trial. Cancer 1977;39:2772-82. \\ 4 Shapiro S, Venet W, Strax P, Venet L, Roeser R. Ten- to fourteen-year effect of breast cancer screening on mortality. Fournal of the National Cancer Institute 1982;69:349-59. \\ 5 Tabar L, Gad A, Holmberg LH, et al. Reduction in mortality from breast cancer after mass screening with mammography. Randomised trial from the Breas Cancer Screening Working Group of the Swedish National Board of Health and Welfare. Lancet 1985;i:829-32. \\ 6 Knox EG. Computer simulations of cervical cytology screening programmes. In:McLachlan G, ed. Future- and present indicatives. Problems and progress in medical care. London: Oxford University Press (for the Nuffield Provincial Hospitals Trust), 1973:30-55. \\ Knox EG Simulation studies of breast cancer screening programmes. In McLachlan G, ed. Probes for health. London: Oxford University Press (for the Nuffield Provincial Hospitals Trust), 1975: 14-44. \\ $8 \mathrm{Knox}$ EG. Ages and frequencies for cervical cancer screening. $\mathrm{Br} \mathrm{J}$ Cancer 1976;3:444-52 \\ (Accepted 23 May 1988}

\title{
Outcome of untreated infection with Entamoeba histolytica in homosexual men with and without HIV antibody
}

\author{
Erica Allason-Jones, Adrian Mindel, Peter Sargeaunt, David Katz
}

Academic Department of Genito-Urinary Medicine and Bland-Sutton Institute of Pathology, University College and Middlesex School of Medicine, London WIN 8AA Erica Allason-Jones, MRCP, lecturer

Adrian Mindel, MRCP, senior lecturer

David Katz, MRCP, reader in experimental pathology

Department of Medical Protozoology, London School of Hygiene and Tropical Medicine, London WC1E 7HT

Peter Sargeaunt, senior chief medical laboratory scientific officer

Correspondence and requests for reprints to: $\mathrm{Dr}$ Allason-Jones.

\begin{abstract}
Among homosexual men the prevalence of infection with Entamoeba histolytica is high. To determine the clinical importance of this infection 55 homosexual men carrying the parasite were investigated in detail. No clinical, serological, or histological evidence of invasive amoebiasis was found in any of them. The patients were not treated and were followed up for 12 to 29 months (mean 21.6 months), during which period none developed symptoms that could be attributed to $E$ histolytica. Spontaneous loss of the parasite occurred in 17 patients, some of whom later became reinfected. Sixteen patients had antibody to human immunodeficiency virus, and infection with $E$ histolytica showed the same benign course in them as in the patients who did not have antibody. Throughout the study classification of the isolates of $E$ histolytica consistently showed that they belonged only to non-pathogenic zymodemes. The findings provide further evidence that $E$ histolytica in homosexual men is a commensal organism.
\end{abstract}

\section{Introduction}

Sexual transmission of Entamoeba histolytica has led to a high prevalence of infection among homosexual men. ${ }^{1-12}$ Several studies have been unable to find any correlation between gastrointestinal symptoms and the presence or absence of $E$ histolytica, ${ }^{136791113}$ and clinical investigations in a few infected men have shown no evidence of invasive disease..$^{1214}$ is Reports that describe invasive amoebiasis in homosexual men are extremely rare. ${ }^{16-19}$

The introduction of zymodeme classification (the grouping of isolates according to their isoenzyme pattern on electrophoresis) has provided a rational explanation for the lack of clinical disease in many people infected with $E$ histolytica. Twenty two different zymodemes of $E$ histolytica have been shown, 12 of which have never been associated with invasive amoebiasis and have therefore been designated non- pathogenic. ${ }^{20-29}$ Classification of isolates of $E$ histolytica from homosexual men in the United Kingdom and North America has to date shown them all to belong to non-pathogenic zymodemes. ${ }^{511141530}$ Information on the natural course of infections with non-pathogenic $E$ histolytica is scant. In a study that followed up 15 subjects passing cysts of $E$ histolytica, some of which were known to be non-pathogenic, all subjects lost the parasite within 18 months. ${ }^{31}$ No such study has been carried out on homosexual men, a group in which the high prevalence of infection with human immunodeficiency virus (HIV) and consequent immune suppression are added complications. We therefore investigated a cohort of 55 homosexual men known to be infected with $E$ histolytica for evidence of invasive amoebiasis and followed them up without treatment to establish the natural course of the infection.

\section{Patients and methods}

We identified patients by random screening, ${ }^{11}$ screening during the investigation of gastrointestinal symptoms, and screening sexual partners of men known to be infected with $E$ histolytica. We took a full history from each patient and gave them a general examination. A proctoscope was passed, and specimens were taken for culture for Neisseria gonorrhoeae, Chlamydia trachomatis, and herpes simplex virus. A blood sample was taken to test for antibodies to amoebas and HIV. Faecal specimens were examined for parasites, including cryptosporidium, and were cultured for salmonellas, shigellas, campylobacter, and yersinia. After these additional infections had been treated or excluded patients who had no contraindication and agreed to the procedure underwent sigmoidoscopic examination, during which two rectal biopsy specimens were taken, one for histological examination and the other for culture of amoebas.

To determine the natural course of infection with $E$ histolytica we did not treat the patients but reassessed 